\title{
Searching for Wolf-Rayet Stars in M101
}

\author{
J. L. Bibby ${ }^{1}$, P. A. Crowther ${ }^{2}$, A. F. J. Moffat ${ }^{3}$, M. M. Shara ${ }^{1}$, \\ D. Zurek $^{1}$ and L. Drissen ${ }^{3}$ \\ ${ }^{1}$ Dept. of Astrophysics, American Museum of Natural History \\ Central Park West @ 79th St, New York, NY 10024, USA \\ email: jbibby@amnh.org \\ ${ }^{2}$ Dept. of Physics \& Astronomy, University of Sheffield, \\ Hounsfield Rd, Sheffield, S3 7RH, UK \\ ${ }^{3}$ Dépt. de physique, Université de Montréal, C.P. 6128, \\ Succursale Centre-Ville, Montréal, QC, H3C 3J7, Canada
}

\begin{abstract}
Wolf-Rayet (WR) stars are the evolved descendants of massive O-type stars and are considered to be progenitor candidates for Type Ib/c core-collapse supernovae (SNe). Recent results of our HST/WFC3 survey of Wolf-Rayet stars in M101 are summarised based on the detection efficiency of narrow-band optical imaging compared to broad-band methods. We show that on average $42 \%$ of WR stars, increasing to $\sim 85 \%$ in central regions, are only detected in the narrow-band imaging. Hence, the non-detection of a WR star at the location of $~ 10$ Type $\mathrm{Ib} / \mathrm{c} \mathrm{SNe}$ in broad-band imaging is no longer strong evidence for a non-WR progenitor channel.
\end{abstract}

Keywords. stars: Wolf-Rayet, galaxies: M101, Survey.

\section{Introduction}

Wolf-Rayet (WR) stars are evolved massive O-type stars which are predicted to be the progenitors of Type Ibc core-collapse supernovae (ccSNe). However, to date there has been no direct confirmation of the WR-SNe connection. Pre-SNe broad-band images have failed to reveal the progenitor of $~ 10$ Type Ib/c SNe (Smartt 2009).

WR stars can be classified into two main subtypes, nitrogen-rich (WN) and carbonrich (WC) stars, which reveal the products of CNO burning and triple alpha reactions, respectively. WR stars exhibit a unique emission line spectrum which is dominated by He II $\lambda 4686$ emission lines for WN stars while WC spectra, which also show weaker He II $\lambda 4686$, are dominated by C III $\lambda 4650$ and C IV $\lambda 5808$ (Crowther 2007). These strong emission lines can be 0.2-2.5 magnitudes brighter than the adjacent continuum, making WR stars easy to detect using narrow-band imaging techniques.

Type Ib SNe are hydrogen-poor, while Type Ic SNe are both hydrogen- and heliumpoor. The similarities between the observed Type Ib and Ic SN spectra and the chemical composition of the WN and WC stars makes them strong progenitor candidates, respectively.

\section{M101}

M101 is a grand-design spiral galaxy which lies face-on at a distance of $6.2 \mathrm{Mpc}$ (Shappee \& Stanek, 2011). It has a high star-formation rate of at least $\sim 4.5 \mathrm{M}_{\odot} \mathrm{yr}^{-1}$ based on the $\mathrm{H} \alpha$ flux or $\sim 6.8 \mathrm{M}_{\odot} \mathrm{yr}^{-1}$ from far UV imaging (Lee et al. 2009).

There is a wealth of HST/ACS archival data for M101 which was obtained under the legacy program, however the most effective way to identify WR stars is via narrow-band 


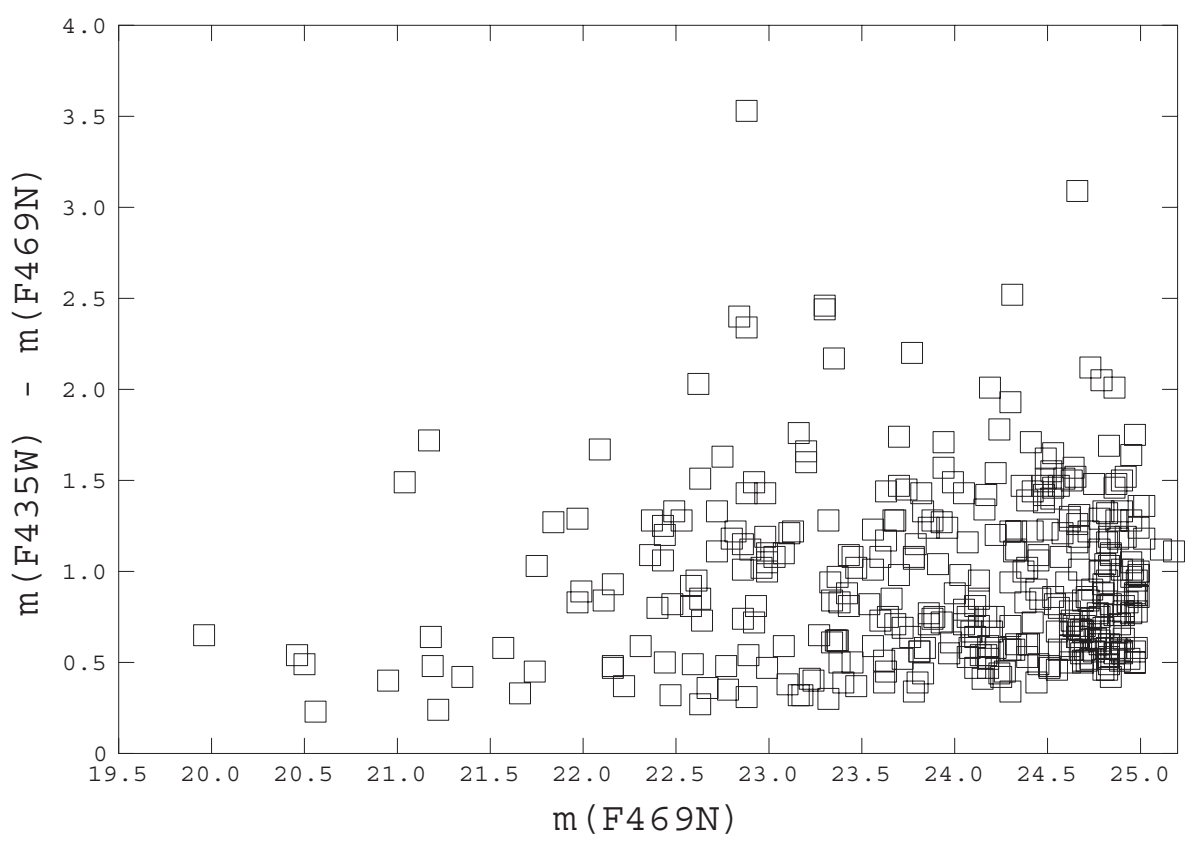

Figure 1. F469N magnitude versus excess magnitude relative to the F435W image for the 372 WR candidates identified in four of the HST/WFC3 pointings.

imaging techniques centered on the WR emission lines. We obtained 18 pointings of M101 using WFC3/F469N over 36 orbits in cycle 17 (PI: Shara).

Identifying WR stars beyond the Local Group with ground-based imaging is challenging since we can only resolve them on scales of $\sim 25 \mathrm{pc}$. The emission lines of WR stars, although strong, can easily be diluted by strong continuum from other OB stars in the unresolved region (Bibby \& Crowther 2010). The high spatial resolution images allow us to resolve sources down to $\sim 3 \mathrm{pc}$, decreasing the contamination of the WR emission by continuum sources.

Typically WR stars span an absolute magnitude range of $\mathrm{M}_{V}=-4$ to -8 mag; however, our ground-based studies do not extend fainter than $\mathrm{M}_{V}=-5 \mathrm{mag}$. The improved sensitivity of HST allows us to identify the faintest and least massive WR stars, detecting stars to $\mathrm{M}_{F 469 N}=-3.5 \mathrm{mag}$ and $\mathrm{M}_{F 435 \mathrm{~W}}=-4 \mathrm{mag}$.

\section{Identifying candidates}

The ACS and WFC3 data were re-drizzled onto the same scale of 0.05 arcsec pix ${ }^{-1}$ using MULTIDRIZZLE. Photometry was performed with the stand-alone DAOPHOT package. Stars that had at least a 3 sigma excess in the F469N filter compared to the F435W and F555W filters were identified as WR candidates. These candidates were then visually inspected using the "blinking" method (Moffat \& Shara, 1983).

Four of the WFC3/F469N pointings have currently undergone analysis and have revealed 372 WR candidates with a He II $\lambda 4686$ excess of at least 3 sigma (Fig. 1). The brightest candidates with $\mathrm{m}(\mathrm{F} 435 \mathrm{~W})-\mathrm{m}(\mathrm{F} 469 \mathrm{~N})$ excess of $\leqslant 2.5$ mag are likely to be single WR stars, whereas the fainter candidates at $\mathrm{m}(\mathrm{F} 469 \mathrm{~N}) \sim 25 \mathrm{mag}$ with small $\mathrm{m}(\mathrm{F} 435 \mathrm{~W})$ $\mathrm{m}(\mathrm{F} 469 \mathrm{~N})$ excesses $\leqslant 0.6 \mathrm{mag}$ are more likely to host multiple WR stars in unresolved clusters. 


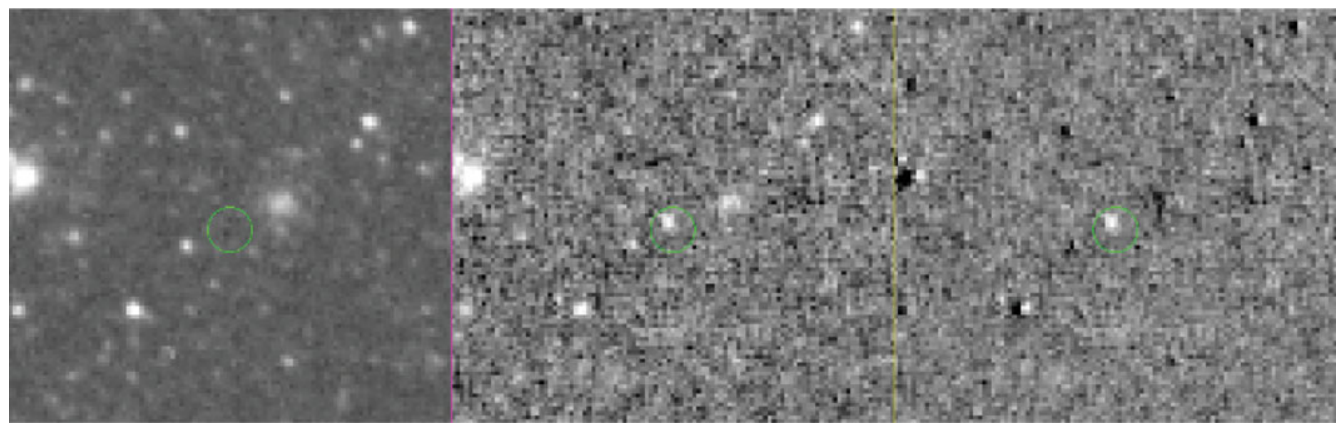

Figure 2. Postage stamp image $(\sim 30 \times 30$ arcsec $)$ of a WR candidate in M101. The F435W broad-band continuum image (left) and narrow-band F469N image (centre) are subtracted to produce the "net" image (right), revealing He II $\lambda 4686$ emission indicating the presence of a WR star.

From the photometric analysis we were also able to identify sources in the F469N image that were not identified in either the $\mathrm{F} 435 \mathrm{~W}$ or $\mathrm{F} 555 \mathrm{~W}$ images. This is indicative of a faint WR star with a continuum which lies below the detection threshold of the broad-band images. We identified an additional 269 WR candidates that were only detected in the narrow-band images, which were again checked using the blinking method (Fig. 2).

We are currently awaiting execution of follow-up multi-object spectroscopy with GeminiNorth/GMOS for a sample of these candidates ( $\sim 35 \%)$ which will allow us to (i) determine the multiplicity of the sources, (ii) assign a spectral classification of WN or WC to the candidate and (iii) infer the subtype of the remaining candidates for which we did not obtain spectroscopic confirmation.

\section{Conclusions \& Future Work}

We identified 641 WR candidates within four HST/WFC3 pointing of M101, $42 \%$ of which are only detected in the narrow-band image. This is much higher than the $25 \%$ found from ground-based studies of other nearby spiral galaxies, e.g. Bibby \& Crowther (2012), which is most likely due to the improved sensitivity and spatial resolution of HST which allows us to detect fainter WR stars. This work highlights the effectiveness of high spatial resolution narrow-band observations in detecting WR stars. If we hope to confirm or, equally important, rule out WR stars as the progenitors of Type Ib/c SNe, then we require a complete sample of WR stars in several nearby galaxies.

Once analysis of M101 is complete we will be able to;

(i) Investigate how the number of WR stars varies with the number of $\mathrm{O}$ stars and Red Supergiants (RSG) across the galaxy as a function of metallicity and compare the results to predictions from theoretical evolutionary models.

(ii) Degrade our HST imaging and re-run the analysis to determine how many WR stars are not detected, and hence estimate the true completeness of our ground-based surveys.

(iii) Analyse the properties of the regions associated with WR stars to assess whether they are consistent with Type Ib/c SNe.

(iv) Expand our existing catalogue of WR stars which can then be referred to in the event that a Type Ib/c SN occurs in one of these galaxies. This will allow strong, direct observational evidence for, or against, the WR-SNe connection. 


\section{References}

Bibby, J. L. \& Crowther, P. A. 2012, MNRAS, 420, 3091

Bibby, J. L. \& Crowther, P. A. 2010, MNRAS, 405, 2737

Crowther P. A. 2007, ARAESA, 54, 177

Crowther, P. A. \& Hadfield L. J. 2006, A\&A, 449, 895

Lee, J. C., Gil de Paz, A., Tremonti, C., Kennicutt, R. C. Jr., Salim, S., Bothwell, M., Calzetti, D., Dalcanton, J., et al. 2009, ApJ, 706, 599

Moffat A. F. J. \& Shara, M. M. 1983, ApJ, 273, 544

Shappee, B. J. \& Stanek, K. Z. 2011, ApJ, 733, 124

Smartt, S. J. 2009, ARA\&SA, 47, 63

\section{Discussion}

J. SHIODE: What contaminating sources might masquerade as WR stars?

J. BibBY: We worried about contamination from planetary nebulae, however our detection limits are not deep enough to detect PNe.

J. SHIODE: Can evolutionary calculations really be considered predictions for $\mathrm{N}(\mathrm{WR}) /$ $\mathrm{N}(\mathrm{O})$ given the uncertainty in very massive star mass-loss histories?

J. BIBBY: $\mathrm{N}(\mathrm{WR}) / \mathrm{N}(\mathrm{O})$ ratios have been relatively consistent with observations where we have a complete WR sample. We should not blame the models until we are sure we are detecting all of the WR population. Our M101 survey will detect the faintest WR stars hence our ratios should be reliable and so if they don't match predictions it gives theoreticians something to work with.

S. Chakraborti: What if some of the stars are just variables?

J.BIBBY: We have not encountered such a problem in our ground-based surveys but if $\mathrm{O}$ stars can vary by up to a magnitude on timescales of a few years then this is something we will need to look into. If our follow-up spectroscopy is contaminated by such stars then clearly it is a problem and we need to look at the properties of these stars to see if we can distinguish them from our WR candidates.

T. L. Astrantmadja: Why not look for the WR stars in our Galaxy?

J. BIBby: There are indeed ongoing WR surveys of the Milky Way. However due to the dust extinction in our galaxy IR rather than optical techniques are used. The overall aim of our work is to identify a future SN progenitor, so surveying many nearby galaxies increases our chances. We select our galaxies based on several criteria, one of which is a face-on orientation to avoid the problem of high extinction.

T. L. Astrantmadja: Do WR stars in other galaxies have the same characteristics?

J. BIBBY: Yes, they still have strong emission lines, however the strength of the lines vary with the metallicity of the environment, i.e. in a low metallicity environment the line flux will be reduced as was demonstrated by Crowther \& Hadfield (2006).

P. Mazzali: Can you distinguish from binary WR stars in your survey?

J. BIBBY: Unfortunately we only obtain one epoch of spectra for a sample of WR stars so we cannot identify which stars are in a binary system from radial velocity motion. 